\title{
Optical coherence tomography for detection of compound action potential in Xenopus Laevis sciatic nerve
}

\author{
Francesca Troiani $^{\mathrm{a}}$, Konstantin Nikolic ${ }^{\mathrm{a}}$, and Timothy G. Constandinou ${ }^{\mathrm{a}}$ \\ ${ }^{a}$ Centre for Bio-inspired technology, Dept. of Electrical and Electronic Engineering, \\ Imperial College London, SW7 2AZ, United Kingdom
}

\begin{abstract}
Due to optical coherence tomography (OCT) high spatial and temporal resolution, this technique could be used to observe the quick changes in the refractive index that accompany action potential. In this study we explore the use of time domain Optical Coherence Tomography (TD-OCT) for real time action potential detection in ex vivo Xenopus Laevis sciatic nerve. TD-OCT is the easiest and less expensive OCT technique and, if successful in detecting real time action potential, it could be used for low cost monitoring devices. A theoretical investigation into the order of magnitude of the signals detected by a TD-OCT setup is provided by this work. A linear dependence between the refractive index and the intensity changes is observed and the minimum SNR for which the setup could work is found to be $S N R=2 \times 10^{4}$.
\end{abstract}

Keywords: Optical Coherence Tomography, nerve, intrinsic optical properties

\section{INTRODUCTION}

Since its discovery in the eighteenth century, electrophysiology has always been the golden standard for neural recordings. The signal observed with an electrode in close proximity of the studied tissue has an extremely high temporal and spatial resolution, and it has allowed scientists to improve our understanding of the brain. Nonetheless the use of neurons electrical signals, however extremely useful and precise, is limited by the necessity of the electrodes to be close to the neurons themselves and time has come when the need of less invasive techniques is compelling. Various non-invasive techniques have been developed and are currently widely used, i.e. electroencephalogram and MRI, but their temporal and spatial resolutions are very poor and they cannot be used to study the quick and localised changes that happen when neurons activate.

To overcome this difficulty, the use of optical techniques to study neural activity has been considered. Light, as known, can travel through matter and has been used for a long time to observe and study samples. Measurements of nerve activity using light have been tried in the $1950 \mathrm{~s}^{1}$ and, in $1968 \mathrm{Cohen}^{2}$ proved the existence of a correlation between the change in the electrical activity of different types of non myelinated axons (nerve bundle from the walking legs of Maia squinado crab and the giant axon from the Loligo forbensi squid) and two different optical quantities: birefringence and scattering. However, the changes in these properties resulted extremely small ( $\sim 1 \times 10^{-5}$ for the nerve and $\sim 1 \times 10^{-6}$ for the axon) for scientists to use them to observe neural activity with the technology of the time. Different approaches have been tried, and for the following 40 years research has focused on different - and easier to detect - extrinsic optical quantities, such as voltage sensitive dyes or genetically encoded calcium indicators emitting light during action potentials. ${ }^{3}$

Recently, with the advent of more precise detectors and the development of new interferometric techniques, the interest in the study of intrinsic optical changes has risen again. In this paper, the use of time domain optical coherence tomography to detect neural activity will be discussed.

Further author information: Francesca Troiani, E-mail: f.troiani14@imperial.ac.uk 


\subsection{Optical coherence tomography}

Optical Coherence Tomography (OCT) has been first described in 1991 by Huang et al. ${ }^{4}$ Like ultrasound imaging relies on the echo time and amplitude of a sound wave echo, OCT relies on the same characteristic of reflected light. However, since light speed is too high to allow echo times to be measured directly, the use of low-coherence interferometry is required. Low-coherence interferometry had its first biological applications in ophthalmology ${ }^{4,5}$ and since then had played an important role in medical imaging.

From interference theory, the photodetected signal is: ${ }^{6}$

$$
I_{p d}=\alpha \frac{P_{0}}{2}\left[O+R+2 \sqrt{O R} \Pi \cos \left(\frac{2 \pi}{\lambda} d\right)\right],
$$

where $\alpha$ is the photodetector responsivity, $P_{0}$ the incident power, $\mathrm{O}$ and $\mathrm{R}$ the target and reference mirror reflectivities, $\Pi$ the polarization degree (i.e. the degree of similarity in the orientation of the electric fields of the two light beams), $\lambda$ is the central wavelength of the light source and $d$ is the difference in the optical path the two beams have travelled. In this equation the first two terms are constant in time and the third term is the one describing the interference.

In the case of purely monochromatic waves, i.e. waves whose frequency spectrum can be described by a Dirac delta, the interference signal presents an infinite number of peaks and it repeats itself every time the reference mirror is moved by an extra $\frac{\lambda}{2}$. On the other hand, if the waves are not monochromatic, the photodetected signal will be given by a superposition of Equation 1 for each frequency of the wave. This superposition leads to a sum of cosine waves and a cancellation of the signal for most of the values of $d$. This means that, for a signal to be detected by the photodetector in the case of polychromatic waves, the optical path difference must be close to zero. The "closeness" is given by the coherence length of the light: the more broadband the light source is, the shorter the optical path difference should be.

For a Gaussian spectrum (i.e. for a spectrum in which the frequencies are distributed Gaussian-like), the coherence length is related to the central wavelength $(\bar{\lambda})$ and the spectral width $(\Delta \lambda)$ of the light source: ${ }^{7}$

$$
L_{C}=\frac{4 \ln 2}{\pi} \frac{\bar{\lambda}^{2}}{\Delta \lambda}
$$

This characteristic is fundamental in optical coherence tomography. Using a light source with low coherence length it is possible to increase the depth (axial) resolution $\Delta z:^{8}$

$$
\Delta z=\frac{L_{C}}{2}=\frac{2 \ln 2}{\pi} \frac{\bar{\lambda}^{2}}{\Delta \lambda}
$$

The transverse resolution, as for confocal microscopy, is determined by the beam focusing parameters: ${ }^{8}$

$$
\Delta x=\frac{4 \lambda}{\pi} \frac{f}{d_{s}}
$$

where $f$ is the lens focal length and $d_{s}$ the spot size on the lens. However, the axial and the transverse resolution are correlated and increasing the transverse leads to a decrease in the axial.

An interesting aspect of this technique is that, even if the sample reflectivity is just of $1-2 \%$, the recombination of the sample beam with the reference one boosts the signal, allowing for its detection.

\section{METHODS}

With this study, we want to establish the feasibility of the use of time domain optical coherence tomography to observe neural activity. Time domain OCT is indeed the easiest and less expensive OCT setup; if effective in detecting neural activity, it could lead to low cost non-invasive monitoring devices. However, being much less sensitive than other OCT techniques, a simulated study is necessary before being able to build a setup that allows for detection of the changes in backscattered light due to neural activity. 


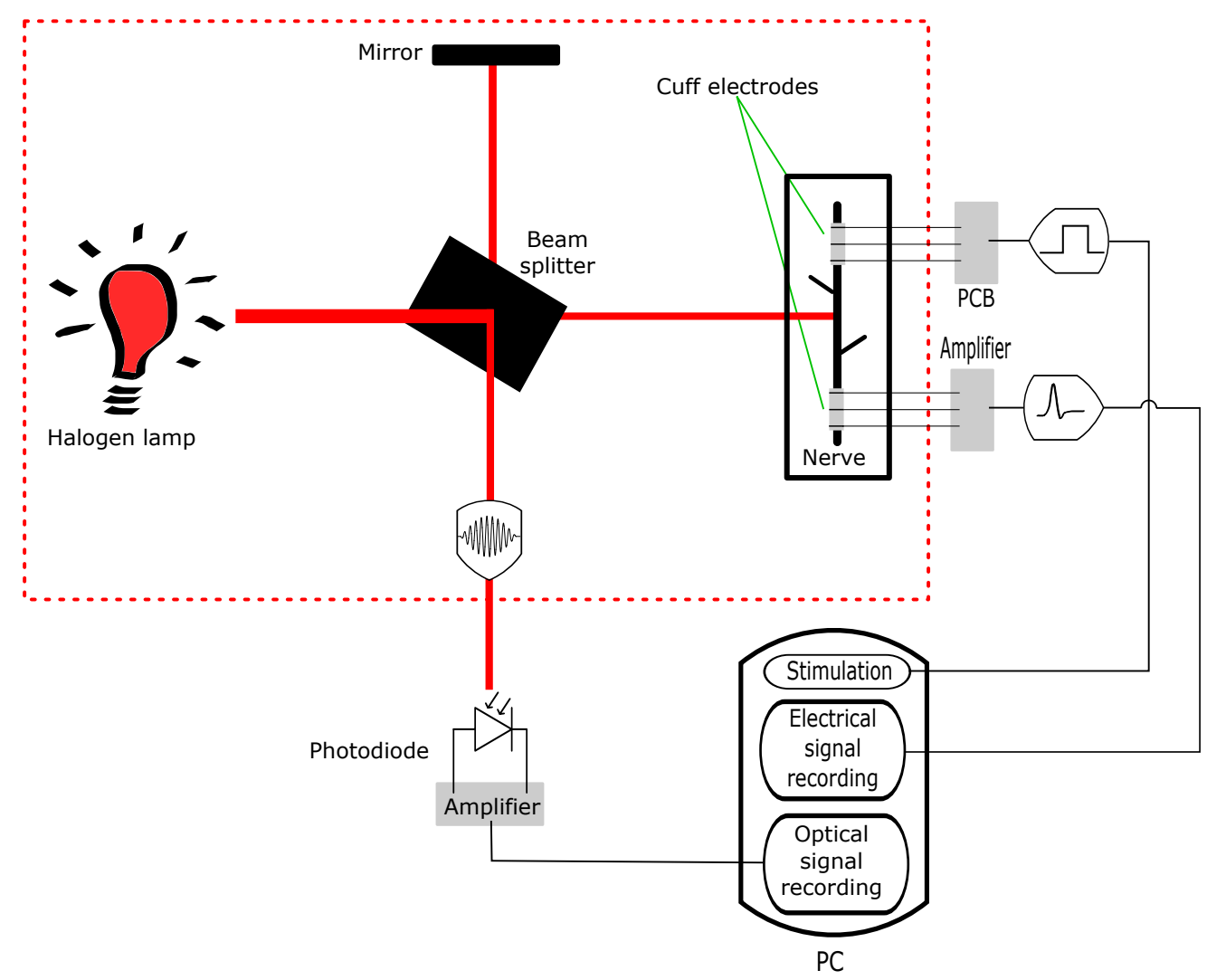

Figure 1. Concept of a possible experimental setup with time domain OCT and electrical recording/stimulation of the nerve.

\subsection{Biological model}

It has been proved that the optical signal detected from the axons has a lower noise than the one detected from somas since somas contain different pigmented and granular organelles causing a background scattering that could bias the measurement. ${ }^{9}$ For this reason, the biological model chosen for this study is a peripheral nerve and, more precisely, a Xenopus laevis sciatic nerve. Inside a nerve, axons are covered by loose connective tissue (Endonerium) and bundled together into fascicles by another layer of connective tissue (Perineurium). These fascicles are held together by a third layer of connective tissue (Epineurium) that creates the nerve surface.

The most important characteristic of the chosen model is that it is made of only one fascicle of axons, therefore it allows for reduction of noise due to multiple connective tissue layers.

Figure 1 shows a possible experimental setup where the nerve is activated using cuff electrodes and its activity is recorded using both cuff electrodes and OCT. The light source chosen for this setup, is a halogen lightbulb. This choice has been made for two different reasons, the first one being related to the decision of trying to build a low cost setup and the second one to the broadness of this kind of sources. However, since AR coatings are available for a limited range of wavelengths, a filter should be applied to limit the range between $600 \mathrm{~nm} \leq \lambda \leq$ $1000 \mathrm{~nm}$.

\subsection{Simulations}

To study the order of magnitude of the changes in the interference pattern for a time domain OCT, simulations have been done using COMSOL Multiphysics and, specifically, its optical rays module. ${ }^{10}$ With this module it is possible to model electromagnetic wave propagation for systems in which the smallest geometric detail is much bigger than the wavelength considered. It is also possible to compute the intensity, phase and polarization of light. 
The simulated setup is shown in Figure 1, enclosed by the red dashed line, and is composed by a light source, a beam splitter, the nerve, a reference mirror and a detector.

\section{RESULTS}

In an ideal setup, where there are no losses, the intensity of the interference pattern detected by the photodetector is given by Equation 1. With the parameters shown in Table 1 the theoretical power at the photodiode for a non active nerve would be $I_{P D}^{n a}=30.25 \mu \mathrm{W}$. If we assume the reflectivity of the nerve would change by $1 \times 10^{-5}$, the theoretical power at the photodiode for an active nerve would then have a $9 \times 10^{-5}$ change.

Table 1. Parameters used for theoretical computation.

\begin{tabular}{c|c|c|c|c|c|c}
$\alpha$ & $P_{0}$ & $\mathrm{O}$ & $\mathrm{R}$ & $\Pi$ & $\mathrm{d}$ & $\lambda$ \\
\hline 0.5 & $100 \mu W$ & 0.01 & 1 & 1 & 0 & $750 \mathrm{~nm}$
\end{tabular}

\subsection{Simulations}

A polychromatic beam which wavelengths are sampled from a Gaussian distribution $(\bar{\lambda}=750 \mathrm{~nm}$ and $\Delta \lambda=300$ $\mathrm{nm}$ ) is released for each simulation. Successive simulations have been done changing the value of the refractive index of the nerve by one part per million at a time until a total change of 10 parts per million. Since the action potential happens on a millisecond scale, while the light travels through the whole setup in a nanosecond scale, it is possible to run different simulations for each value of the refractive index to have an approximation of the optical change. Each simulation has been repeated and the result averaged over the total number of simulations.

A first set of simulation has been done to study the order of magnitude of the intensity changes. Figure 2 shows how the intensity changes with the refractive index. The values obtained have been fitted with a line $I=m \cdot R I+q$ and the value $\mathrm{m}$ has been reported in the graph with the value corresponding to a $95 \%$ confidence level written in the brackets.

Other simulations have been run to study the maximum noise level of the lightsource for the signal to be still correctly detected. Noise with different standard deviations has been added to the initial intensity of the lightsource $I_{0}$ and the results are shown in Figure 3, where SNR is the ratio between $\left\langle I_{0}\right\rangle$ and the standard deviation of the added noise. As in the previous case, the data have been fitted with a line $I=m \cdot R I+q$. An ANOVA test has been run to see if the intensity value for the inactive nerve (refractive index change $=$ 0 ) and the intensity value for the completely active nerve (refractive index change $10^{-5}$ ) are distinguishable. The $\mathrm{p}$ value of the test resulted less than 0.05 for the first three simulations $\left(p_{1}=2.1 \times 10^{-8}, p_{2}=0.0015\right.$, $p_{3}=0.0388$ for $S N R_{1}=2 \times 10^{5}, S N R_{2}=5.9 \times 10^{4}$ and $S N R_{3}=2 \times 10^{4}$ respectively) but bigger in the one with $S N R_{4}=1.43 \times 10^{4}\left(p_{4}=0.1792\right)$. It is thus possible to say that for a SNR smaller than $2 \times 10^{4}$ it is no more possible to observe the decreasing trend of the data.

\section{DISCUSSION}

Optical coherence tomography is a technique which high spatial and temporal resolution allow for a real time detection of neural activity. The use of phase sensitive OCT has been already proved to be effective to detect activity in squid Loligo pealeii's unmyelinated nerve fibres. ${ }^{11}$ With this study, we wanted to establish if, and to which extent, it is possible to use Time Domain OCT to detect neural activity in a peripheral nerve.

A central wavelength of $\bar{\lambda}=750 \mathrm{~nm}(f=400 \mathrm{THz})$ has been chosen because in soft tissues - where the absorption and scattering coefficients for wavelengths in the range $600-1000 \mathrm{~nm}$ are respectively $\mu_{a}=0.1-10$ $\mathrm{mm}^{-1}$ and $\mu_{s}=10-100 \mathrm{~mm}^{-1}$ - the red end of the visible spectrum provides a window for optical radiation. ${ }^{12}$ For smaller wavelengths $(f>500 \mathrm{THz})$, the absorption coefficient decreases but the scattering one increases while for bigger wavelengths $(f<300 \mathrm{THz})$ the absorption coefficient becomes so big that the penetration depth plummets to only $10^{-2} \mathrm{~cm}$.

Reflections and absorption due to the small layer of water (Ringer solution) that should cover the nerve in an experimental setup, have not been taken into account in this study. In a possible experiment, cold water could 


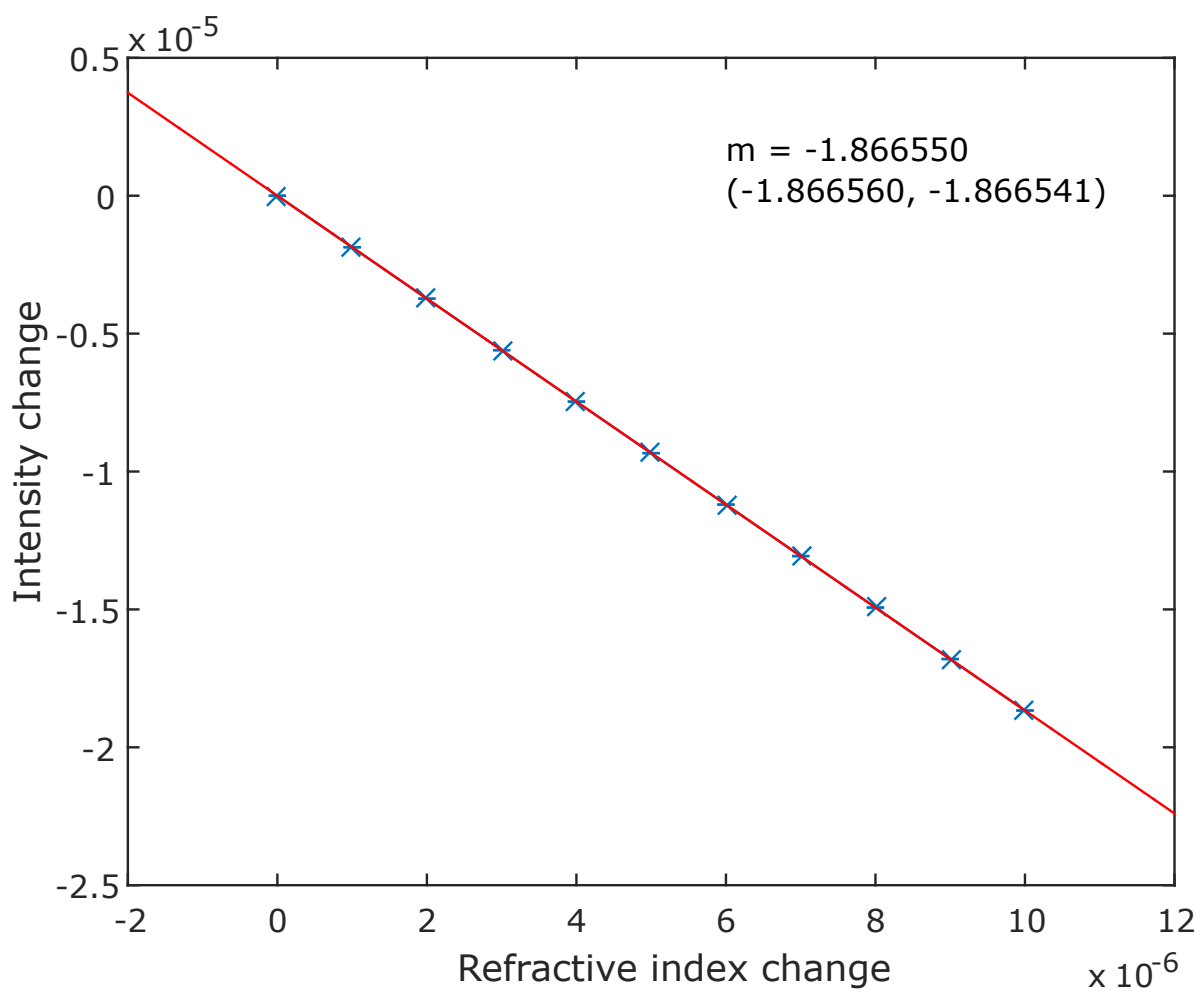

Figure 2. Change in intensity vs change in refractive index.

be used to slow down the action potential and obtain a better time resolution, or the water refractive index could be modified to enhance the signal obtained from the nerve. Only reflections due to the external surface of the nerve have been considered in this paper but, in the future, further studies will be performed to simulate the possibility of the detection of single fibres inside the nerve.

\section{CONCLUSIONS}

The simulations account for the various losses due to multiple reflections, air and AR coating of the beamsplitter, giving results reasonably smaller than the ones obtained by calculations for an ideal environment. The simulated results, with a change of intensity of the order of $2 \times 10^{-5}$ show that it is possible to measure activity with a time domain OCT setup provided the use of a light source which SNR is no smaller than $2 \times 10^{4}$.

\section{ACKNOWLEDGMENTS}

This work has been supported by the UK Engineering and Physical Sciences Research Council (EPSRC).

\section{REFERENCES}

[1] Hill, D. K. and Keynes, R. D., "Opacity changes in stimulated nerve," The Journal of Physiology 108, 278-281 (May 1949).

[2] Cohen, L. B., Keynes, R. D., and Hille, B., "Light scattering and birefringence changes during nerve activity," Nature 218, 438-441 (1968).

[3] Scanziani, M. and Häusser, M., "Electrophysiology in the age of light," Nature 461(7266), 930-939 (2009).

[4] Huang, D., Swanson, E. A., Lin, C. P., Schuman, J. S., Stinson, W. G., Chang, W., Hee, M. R., Flotte, T., Gregory, K., Puliafito, C. A., and Fujimoto, J. G., "Optical coherence tomography," Science 254(5035), 1178-1181 (1991). 

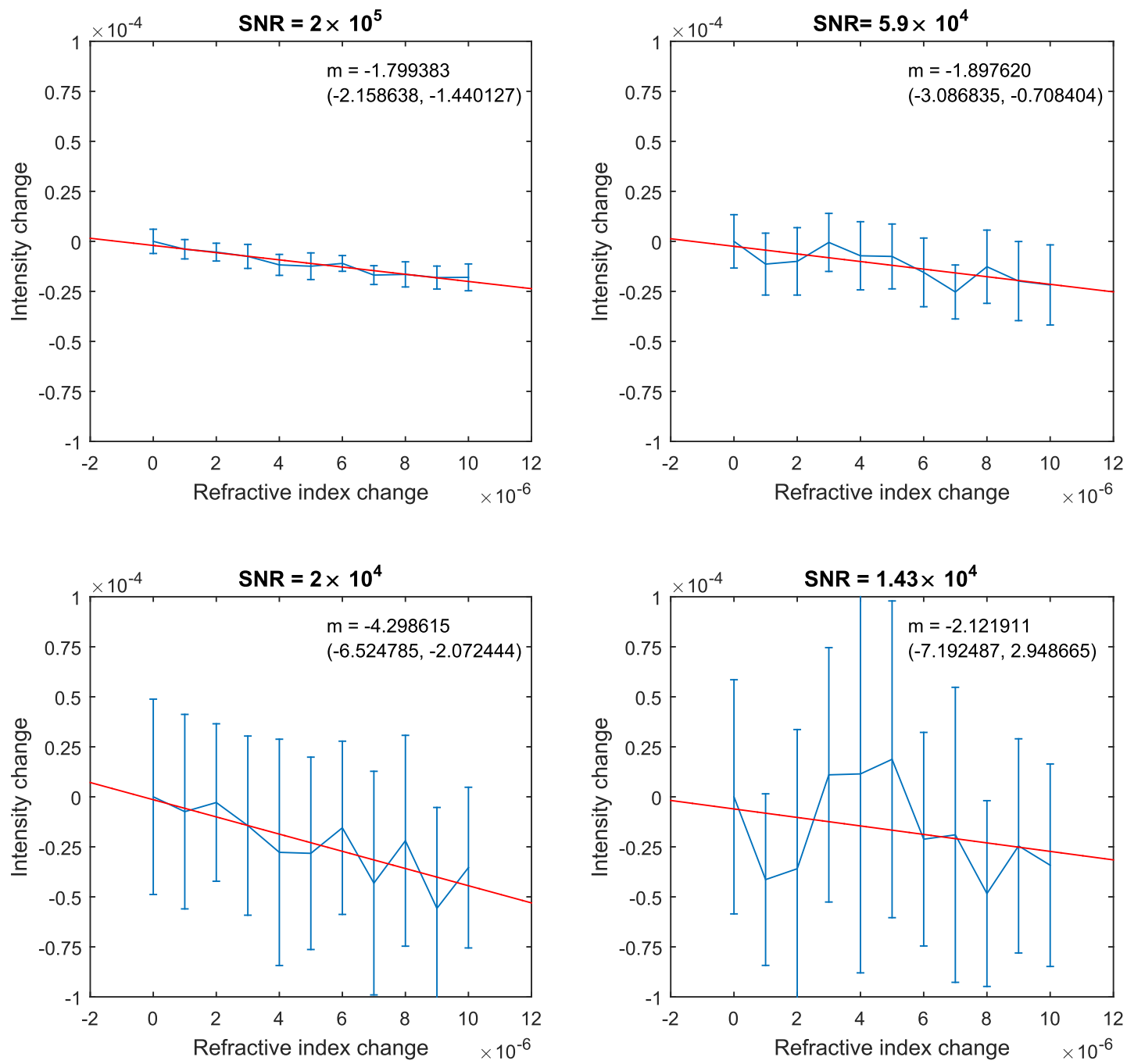

Figure 3. Change in intensity vs change in refractive index for different noise levels at the light source. SNR is the ratio between $\left\langle I_{0}\right\rangle$ and the standard deviation of the added noise. The data are fitted with a line $I=m \cdot R I+q$. In brackets are the value for $\mathrm{m}$ corresponding to a $95 \%$ confidence level. 
[5] Fercher, A. F., Mengedoht, K., and Werner, W., "Eye-length measurement by interferometry with partially coherent light," Optics Letters 13, 186-188 (Mar 1988).

[6] Podoleanu, A. G., "Optical coherence tomography," The British Journal of Radiology 78(935), 976-988 (2005). PMID: 16249597.

[7] Serranho, P., Morgado, A., and Bernardes, R., "Optical coherence tomography: A concept review," in [Optical Coherence Tomography], Bernardes, R. and Cunha-Vaz, J., eds., Biological and Medical Physics, Biomedical Engineering, 139-156, Springer Berlin Heidelberg (2012).

[8] Fujimoto, J., "Optical coherence tomography: Principles and applications," The Review of Laser Engineering 31(10), 635-642 (2003).

[9] Stepnoski, R. A., LaPorta, A., Raccuia-Behling, F., Blonder, G. E., Slusher, R. E., and Kleinfeld, D., "Noninvasive detection of changes in membrane potential in cultured neurons by light scattering.," Proceedings of the National Academy of Sciences of the United States of America 88(21), 9382-9386 (1991).

[10] Comsol Multiphysics, Ray Optics Module, vs 5.2 ed. (2015).

[11] Yeh, Y.-J., Black, A. J., Landowne, D., and Akkin, T., "Optical coherence tomography for cross-sectional imaging of neural activity," Neurophotonics 2(3), 035001 (2015).

[12] Wilson, B. and Jacques, S. L., "Optical reflectance and transmittance of tissues: principles and applications," IEEE Journal of Quantum Electronics 26, 2186-2199 (Dec 1990). 